\title{
Rheumatic Fever and Therapeutic Agents: Analytical Methods for the Determination of the Agents in Biological Fluids
}

\section{Chika J Mbah*}

Department of Pharmaceutical and Medicinal Chemistry, University of Nigeria, Nigeria

*Corresponding author: Chika J Mbah, Department of Pharmaceutical and Medicinal Chemistry, Faculty of Pharmaceutical Sciences, University of Nigeria, Nsukka, Enugu State, Nigeria, Email: chika.mbah@unn.edu.ng

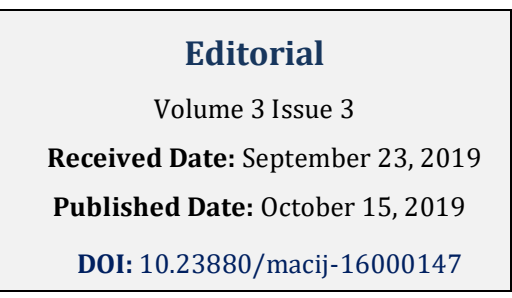

\section{Editorial}

Rheumatic fever is an autoimmune inflammatory response to throat infection caused by streptococcus pyogenes [1]. It may involve connective tissues of the heart, skin and vessels. The disease usually occurs in patients between 5 and 15 years of age. The symptoms of acute rheumatic fever are described by Jones' criteria and patients that meet these criteria are highly likely to have the disease [2-4]. The criteria are divided into major and minor classes.

The major criteria include

- Polyarthritis (usually asymmetrical and migratory but can be additive)

- Carditis (clinical or subclinical and usually manifest as mitral valve regurgitation or aortic valve regurgitation)

- Sydenham's chorea (jerky, uncoordinated movements, especially affecting the face, feet, hands, tongue and the movements disappear during sleep)

- Subcutaneous nodules ( round, firm, freely mobile, painless that occur in crops over the elbows, wrists, knees, ankles etc.)

- Erythema marginatu, (occurs as circular patterns of bright pink macules or papules on the trunk and proximal extremities and not itchy or painful).

The minor criteria are

- Arthralgia (if it occurs in the same pattern as rheumatic polyarthritis)

- Fever
- Elevated acute phase reactants (a serum C-reactive protein level of $\geq 30 \mathrm{mg} / \mathrm{L}$ or erythrocyte sedimentation rate of $\geq 50 \mathrm{~mm} / \mathrm{h}$ )

- Prolonged P-R interval (the P-R interval increases normally with age hence has to be age-adjusted).

Diagnosis of rheumatic fever is highly inferred when two major criteria or one major and two minor criteria are met in a patient with previous streptococcus infection $[5,6]$. Management of rheumatic fever involves prevention and treatment [7]. Prevention could be primary or secondary prevention [8]. In primary prevention, efforts are made to treat the throat infection that precedes rheumatic fever in a timely and effective way using antibiotics such as benzathine penicillin or phenoxymethylpenicillin or amoxicillin. While in secondary prevention, the objective is to stop recolonization or re-infection of the throat with group a streptococci hence preventing recurrence of rheumatic fever. It entails continuous administration of antibiotics (usually parenteral benzathine penicillin) to cases with previous rheumatic fever.

Current clinical treatments of acute rheumatic fever are based on managing symptoms of the disease with therapeutic agents (drugs). Such symptoms and therapeutic agents include:

- Streptococcal infection: amoxicillin, benzathin penicillin, ethromycin ethylsuccinate, penicillin $\mathrm{V}$. 


\section{Medicinal \& Analytical Chemistry International Journal}

- Symptomatic arthritis or arthralgia: acetyl salicyclic acid (aspirin), ibuprofen, naproxen, paracetamol.

- Carditis and other heart diseases: captopril, digoxin, enalapril, furosemide, lisinopril, prednisone (or prednisolone), spironolactone.

- Severe chorea: carbamazepine, valproic acid

Due to the complexity of the disease, various approaches involving use of drugs of different pharmacological classes for effective treatment of symptoms have been utilized. The effective treatment of the symptoms may require administering the drugs as single dosage forms containing two or more drugs or combination of dosage forms having individual drugs. Therefore, accurate, precise, selective, sensitive and specific analytical methods are required in order to accurately quantify the drugs in biological fluids. Biological fluids are very essential to life and help maintain body homeostasis. Whole blood, serum and plasma are the matrices of choice for therapeutic drug monitoring of drugs, however drug concentration can be also measured in saliva, urine, bile, cerebrospinal fluid, tears, pleural fluid etc. Analytical methods mostly employed are spectroscopic and chromatographic methods. However, immunoassay, microbiological assay and capillary electrophoresis methods are also utilized. Amongst the chromatographic methods, high performance liquid chromatography or gas chromatography is mostly used either as hyphenated or non-hyphenated system. Hyphenation is an on-line combination of a chromatographic technique and one or more spectroscopic detection techniques.

In the present article, the objective of the study was to present analytical methods used to determine therapeutic agents involved in the management of the disease in biological fluids. It is envisaged that the information would assist clinicians and pharmaceutical analysts when confronted with the task of ascertaining the levels of any of these drugs in biological fluids.

Although various bioanalytical methods (as previously stated) have been reported for each therapeutic agent, however, only few hyphenated or non-hyphenated chromatographic methods will be presented. They include:

- Amoxicillin (antibiotic) determined in human plasm [9].

- Erythromycin ethylsuccinate (antibiotic) determined in human plasma [10].
- Ibuprofen (anti-inflammatory) determined in human plasma [11].

- Enalipril (anti-hypertensive) determined in human serum [12].

- Digoxin (anti-arrhythimias) determined in human whole blood [13].

- Prednisone (steroidal anti-inflammatory) determined in human serum [14].

- Carbamazepine (anti-epileptic) determined in human plasma [15].

- Valproic acid (anti-epileptic) determined in human saliva [16].

As the bioanalytical methods are not exhaustive, however they demonstrate that these therapeutic agents have been determined accurately in biological fluids. This is very important because effective treatment of disease can be assessed by measuring the drug concentration(s) in the biological fluids.

\section{Conclusion}

Rheumatic fever is a prevalent disease especially in developing countries and rheumatic carditis is the most vital of its clinical manifestations. Treatment of rheumatic fever depends mostly on the symptoms. Antibiotic, antiinflammatory, analgesic, anti-arrhythimia and antiepileptic agents are classes of therapeutic agents widely used in the management of the symptoms. Due to low therapeutic indices of some these therapeutic agents, there is a need to monitor their concentrations in biological fluids during therapy. Chromatographic methods (hyphenated or non-hyphenated) have become the methods of choice in their determinations due to their accuracy, precision, selectivity and sensitivity. However, hyphenation techniques (for example HPLC/MS/MS) have led to very significant improvements in terms of assay sensitivity, specificity and the potential to reduce analysis time and therefore currently the analytical techniques of interest.

\section{References}

1. Karthikeyan G, Guilherme L (2018) Acute rheumatic fever. The Lancet 392(10142): 161-174.

2. Pereira BAF, Belo AR, Silva NAD (2017) Rheumatic fever: update on the Jones criteria according to the America Heart Association review-2015. Rev Bras Rheumatol 57(4): 364-368. 


\section{Medicinal \& Analytical Chemistry International Journal}

3. Beaton A, Carapetis J (2015) The 2015 revision of the Jones criteria for the diagnosis of acute rheumatic fever: implications for practice in low-income and middle-income countries. Heart Asia 7(2): 7-11.

4. Szczygielska J, Hernik E, Kolodziejezyk B, Gazda A, Maslinke M, et al. (2018) Rheumatic fever-New diagnostic criteria. Reumatologia 56(1): 37-41.

5. Webb R, Grant C, Harnden A (2015) Acute rheumatic fever. BMJ 351: h3443.

6. Mahfouz RA, Alawady WS, Salem AS (2017) Ventricular dyssynchrony as a marker of latent carditis in children with acute rheumatic fever: A tissue Doppler imaging. Echocardiography 34(11): 1667-1673.

7. de Barros Branco CE, Orismar Sampaio R, Maia Bracco M, Saady Morhy S, Campos Vieira ML, et al. (2016) Rheumatic fever: A neglected and under diagnosed disease. New perspective on diagnosis and prevention. Arq Bras Cardiol 107(5): 482-484.

8. World Health Organization (2001) Rheumatic fever and rheumatic heart disease: report of a WHO expert consultation. WHO, Geneva, WHO Tech Rep Ser 923.

9. Pingale SG, Badgujar M, Mangaonkar1 KV, Mastorakis NE (2012) Determination of amoxicillin in human plasma by LC-MS/MS and its application to a bioequivalence study. Wseas Transac Biol Biomed 9(1): 1-13.

10. Jangid AG, Tale RH, Vaidya VV (2011) A robust, simple and rapid validated method for estimation of erythromycin ethylsuccinate in human plasma by liquid chromatography tandem mass spectrometry and its application to clinical study. J Pharm Formulation Anal 2(1): 1-7.

11. Szeitz A, Edginton AN, Peng HT, Riggs KW (2010) Assay for the determination of ibuprofen in human plasma using ultra performance liquid chromatography with tandem mass spectrometry (UPLC-MS/MS). Am J Anal Chem 2(2): 47-58.

12. Makwana K, Dhamecha R, Pandya N (2013) Bioanalytical method validation for the determination of enalapril in human serum by LC/MS/MS detection. Indian J Res 2(3): 296-298.

13. Qiestad EL, Johansen U, Opdal MS, Bergan S, Christophersen AS (2009) Determination of digoxin and digitoxin in whole blood. J Anal Technol 33(7): 372-378.

14. Frerichs VA, Tornatore KM (2004) Determination of the glucocorticoids prednisone, prednisolone, dexamethasone, and cortisol in human serum using liquid chromatography coupled to tandem mass spectrometry. J Chromatogr B Analyt Technol Biomed Life Sci 802(2): 329-338.

15. Ezzeldin1 E, Shahat AA, Basudan OA (2013) Development and validation of an HPLC method for the determination of carbamazepine in human plasma. Life Science J 10(4): 2159-2161.

16. Tonic-Ribarska J, Haxhiu A, Sterjev Z, Kiteva G, Suturkova L, et al. (2012) Development and validation of a bioanalytical LC-UV method with solidphase extraction for determination of valproic acid in saliva. Acta Pharm 62(2): 211-220. 